\title{
MIGRATIONS DES POPULATIONS DE GENITEURS DE LA BREME VIMBA (VIMBA VIMBA L.) DANS LE BASSIN DE LA VISTULE
}

\author{
par le docteur STANislas BONTEMPS (1), \\ Chargé de Recherches à l'Institut des Pêches continentales, Pologne.
}

L'espèce Vimba vimba $\mathbf{L}$. est un cyprinidé des rivières de l'Europe de l'Est, voisin de la brême (Abramis brama L.). On le trouve dans l'Elbe en Allemagne, dans la Volga en Russie, en Suède méridionale et dans le Danube. A còté de la forme typique Vimba vimba, il existe des races Vimba vimba carinata (Mer Noire), Vimba vimba persae (Mer Caspienne), Vimba vimba lenella (affluents de la Mer Noire). Cette espèce se caractérise par de grandes migrations de fraie.

En Pologne on trouve l'espèce Vimba (nom polonais : certa) dans les rivières Oder, Warta, Vistule et dans les tout petits affluents de la Mer Baltique.

Dans le bassin de la Vistule (fig. 1), on capture chaque annèe à peu près 300 tonnes de cette espèce, qui a donc une importance économique certaine. Il est nécessaire de ce fait de bien connaitre sa biologie, et notamment ses migrations.

La population est composée de géniteurs qui ont effectué leur croissance dans la mer Baltique (golfe de Gdansk), peu salée (3\% de sel marin);

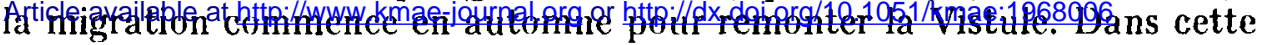
population, les poissons de 6 à 7 ans dominent, mais on en a trouvé âgés de 4 à 9 ans. La longueur des individus est comprise entre 20 et $38 \mathrm{~cm}$, le poids est supérieur à $500 \mathrm{~g}$.

L'espèce Vimba fait alors sa migration clans les affluents de la Vistule pour la ponte qui commence à la fin mai.

\section{BUT DU TRAVAIL}

Les opérations de marquage, entreprises dans la Vistule, ont eu un double but :

1. Apprécier chez les poissons marqués la représentativité de la population de géniteurs, en migration de reproduction.

(1) Le Docteur Bontenps a bien voulu résumer à l'intention du Bulletin français de Pisciculture les recherches qu'il a effectuées et qui ont paru dans les Annales des Sciences agronomiques de Pologne, tome 90, série H, fascicule 4, 1967. 
2. Déterminer :

a) La périorle de migration, l'étendue, la ritesse et les facteus qui influent sur cette vitesse ;

b) L'existence d'une seule population ou de plusieurs populations effectuant des migrations différentes;

c) La présence d'une dispersion possible du troupeau pendant la migration.

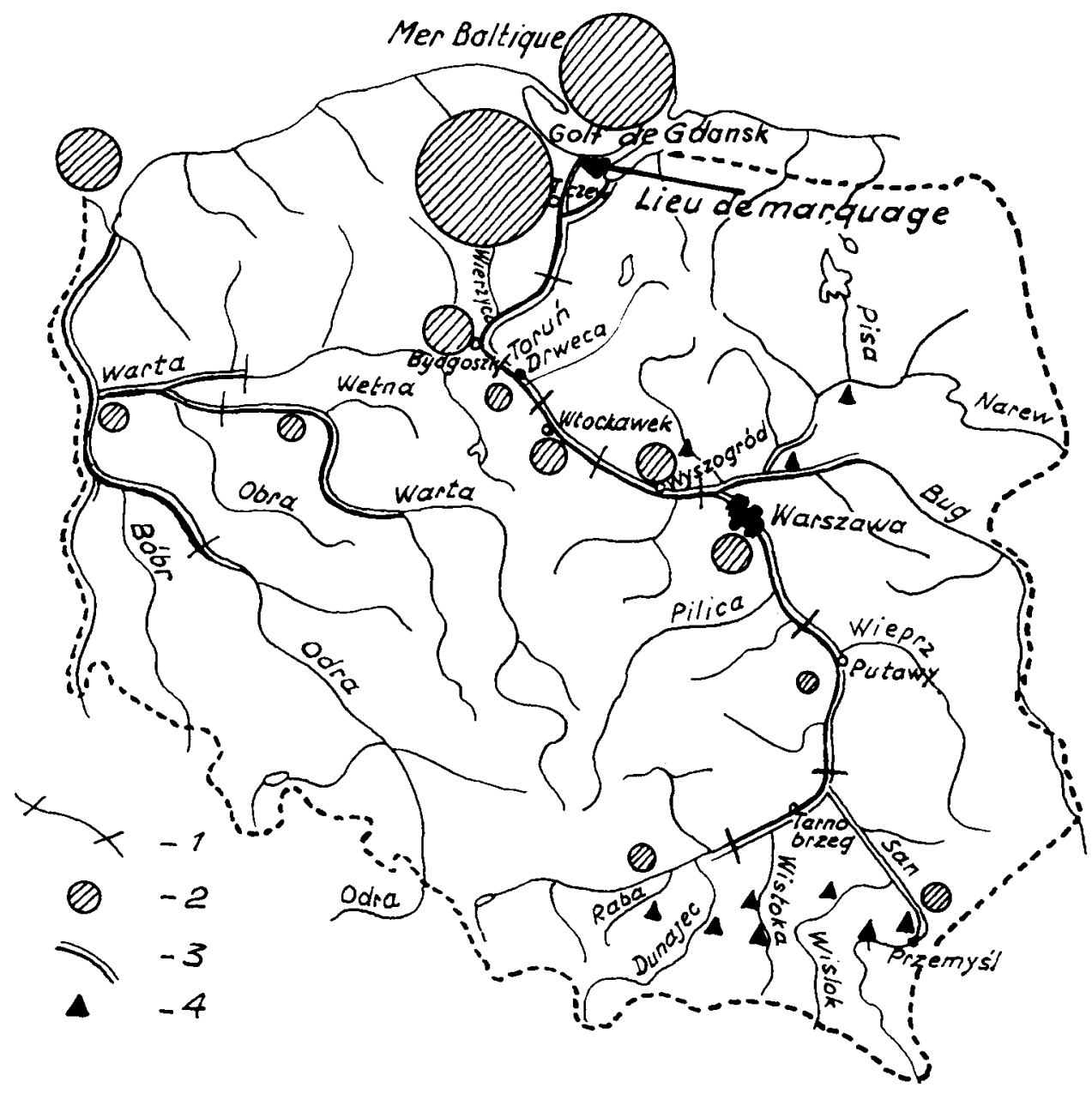

Fig. 1. - Carte de la Pologne

figurant l'étendue des migrations de la Brême l'imba.

1. Emplacements des coopératives de pèche.

2. Nombre relatif de caplures annuelles de Brême Vimba.

3. Rivières où la pèche est professionnelle.

4. Les plus longs relours de marque. 


\section{LES OPÉRATIONS PROPREMENT DITES}

1. Pendant 3 ans (1961-1963), 10.000 poissons ont été marqués dans l'embouchure de la Vistule, à l'époque du commencement de la migration.

2. Les statistiques de pèche de ces poissons ont été tenues pendant 12 ans.

Les poissons étaient marqués à l'aide de marques en celluloïd suspendues par un fil métallique derrière la nageoire dorsale. Le nombre des poissons marqués et le nombre des marques retournées sont données dans la figure 2.

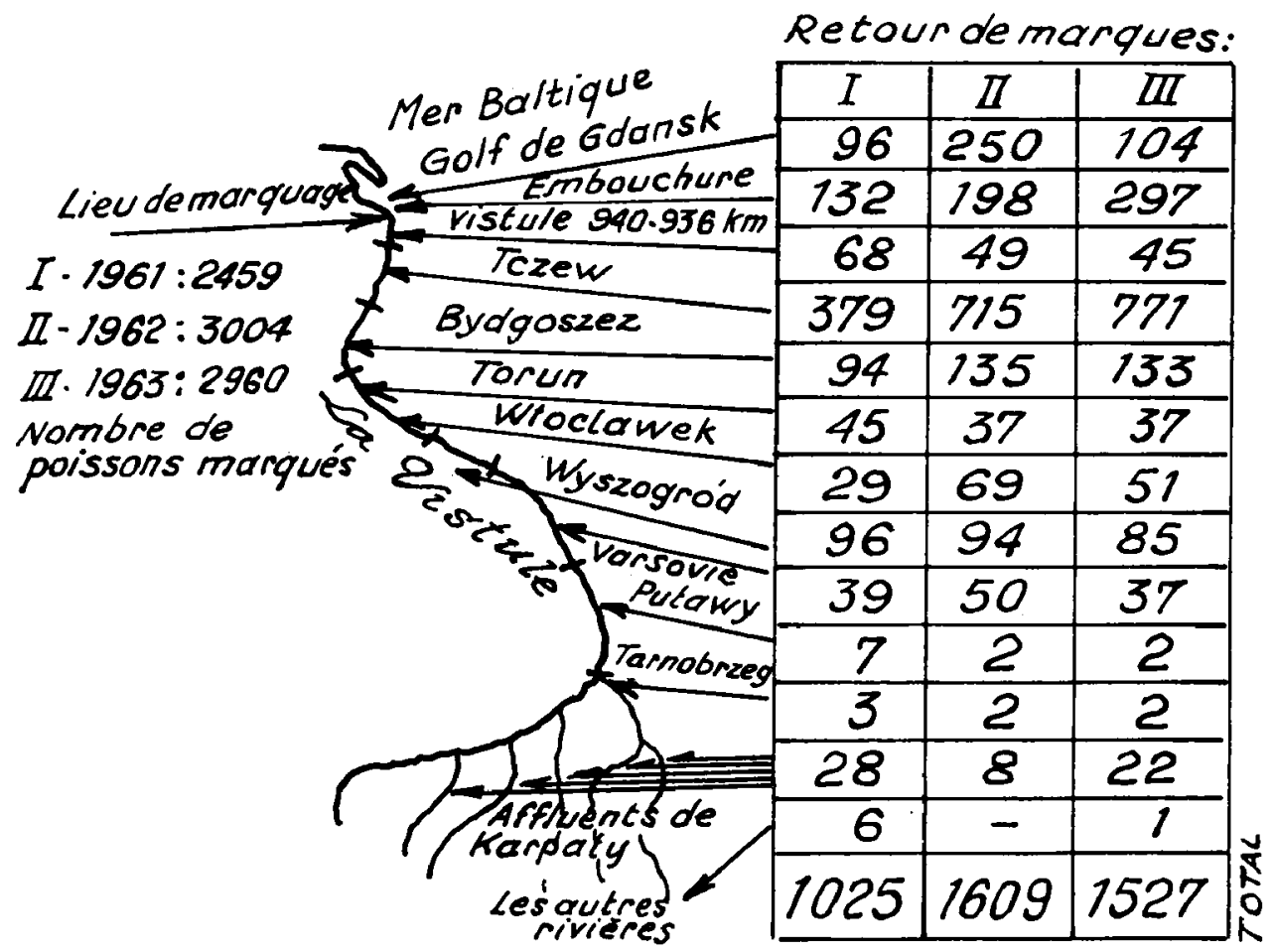

l.ïg. 2. - Nombre de poissons marqués et nombre de marques retournées. 


\section{REPRESENTATIVITÉ DU MARQUAGE}

Puisque la connaissance des migrations de l'espèce l'imba repose sur l'analyse des retours de marques, on considère que les poissons marqués peuvent représenter la population de géniteurs, en migration de reproduction.

On émet l'hypothèse que le nombre de marques retournées doit ètre proportionel au nombre des poissons capturés dans les differentes sections de la Vistule et on calcule le nombre de marques thériques qui devraient correspondre à ces captures pendant la mème période.

labl. 1. -- Retours de marques (en $\%$ ).

\begin{tabular}{|c|c|c|c|c|c|c|}
\hline \multirow{2}{*}{ Régions } & \multicolumn{2}{|c|}{1961} & \multicolumn{2}{|c|}{1962} & \multicolumn{2}{|c|}{1963} \\
\hline & A & B & A & $\mathbf{B}$ & $\mathbf{A}$ & B \\
\hline Tczew & 64,0 & 58,4 & 81,2 & 64,5 & 79,1 & 67,2 \\
\hline Bydgoszcz ... & 11,7 & 13,0 & 6,9 & 12,3 & 7,5 & 12,6 \\
\hline Torun .......... & 4,9 & 6,9 & 1,7 & 3,5 & 2,2 & 3,5 \\
\hline Wloclawek ...... & 2,1 & 4,5 & 2,3 & 6,4 & 3,4 & 4,8 \\
\hline Wyszogrod . & 7,7 & 11,4 & 3,0 & 8,7 & 3,4 & 8,0 \\
\hline Warszawa....... & 4,0 & 4,1 & 3,9 & 4,3 & 3,4 & 3,5 \\
\hline Pulawy .... & 2,8 & 1,2 & 0,4 & 0,1 & 0,4 & 0,2 \\
\hline Tarnobzeg & 3,2 & 0,5 & 0,6 & 0,2 & 0,6 & 0,2 \\
\hline
\end{tabular}

Chaque année on obtenait à peu près les mèmes résultats : au voisinage du lieu de marquage le nombre de retours de marques était moins important que le nombre théorique calculé d'après les poissons capturés (tabl. 1), mais dans des portions de la Vistule situées entre Bydgoszcz et Wyszogrod les retours réels dominaient. La situation changeait dans la partie amont de la Vistule où on trouvait moins de marques vraies (régions de Pulawy et de Tarnobrzeg).

ll convient donc de rechercher les causes de ces divergences qui peuvent ètre les suivantes :

a) Disparition des marques chez une partic des poissons;

b) Les poissons marqués passent inaperçus aux pêcheurs (ce fait est très important au niveau de Tczew où on capture le plus grand nombre (le poissons) ;

c) Les poissons marques sont capturés en plus grand nombre que les poissons non marqués. 
Pour essaver de vérifier comment ces faits conduisent à de lelles divergences on a fait un tableau récapitulatif dans lequel ont été prises en considération toutes les causes indiquées ci-dessus en hypothèse. Ce tableau les a confirmées pleinement.

A côté de ces divergences et aprés leur prise en considération on peut dire que les poissons marqués sont représentatifs du troupeau migrateur.

\section{MIGRATION DE LA FRAIE}

\section{Période de migration.}

Les migrations reproductrices commencent, à l'embouchure des rivières, à la mi-aoùt et durent jusqu’à la mi-octobre.

\section{Rayon de migration.}

Jes plus longs retours de marques ont été constatés dans les affluents de la Vistule : San, Wislok, Wisloka, Dunajee et Raba (fig. 1). A ces endroits on retrouve les plus grandes frayères de cette espèce. Le plus long parcours a été de $856 \mathrm{~km}$.

\section{Vitesse de migration.}

Après les opérations de marquage dans l'estuaire de la Vistule, une partie des poissons se retire en mer (environ $20 \%$ ) et commence sa migration plus tard, à différentes époques. Il en résulte une vitesse apparente différente pour la région voisine de l'estuaire (région de Tczew) qui, pour cette raison, a été exclue de l'étude de la vitesse de migration. Dans le graphique 3 , on a donné la vitesse de migration pendant 3 années successives et obtenu les vitesses moyennes respectives de $6,3,5,5$ et $7,5 \mathrm{~km}$ par jour, soit pour trois années $6,1 \mathrm{~km}$ par jour. Les poissons qui font leur migration à cette vitesse peuvent atteindre les frayères en 4 à 5 mois.

Les temps moyens de migration ont été complètés par l'analyse des groupes cle vitesse.

Dans la figure 3 , nous voyons que la vitesse de migration donne une grande amplitude de $1-2$ jusqu'à $17 \mathrm{~km}$ par jour. On divise le troupeau en groupes de vitesse et on détermine en pourcentage le nombre de géniteurs qui peuvent atteindre les frayères avant l'hiver, au printemps de l'année suivante et de ceux qui ne peuvent arriver à temps pour la prochaine ponte (tabl. 2), on peut dire que 78,9 à $91,5 \%$ du troupeau qui commence sa migration en automne arrive à pondre dans les rivières des Carpathes pendant la période de ponte du printemps suivant (mai, juin). 


\section{$-138-$}

Tabl. 2. - Groupes de vitesse.

\begin{tabular}{|c|c|c|c|}
\hline Année de marquage & $\begin{array}{c}\text { Vitesse } \\
1-2,9 \mathrm{~km} / \text { jour }\end{array}$ & $\begin{array}{c}\text { Vitesse } \\
3-9,9 \mathrm{~km} / \mathrm{jour}\end{array}$ & $\begin{array}{c}\text { Vitesse } \\
10 \mathrm{~km} / \mathrm{jour} \\
\text { et plus }\end{array}$ \\
\hline 1961 & $12,4 \%$ & $73,4 \%$ & $14,2 \%$ \\
\hline 1962 & $21,1 \%$ & $71,4 \%$ & $7,5 \%$ \\
\hline 1963 & $8,5 \%$ & $72,9 \%$ & $18,6 \%$ \\
\hline
\end{tabular}

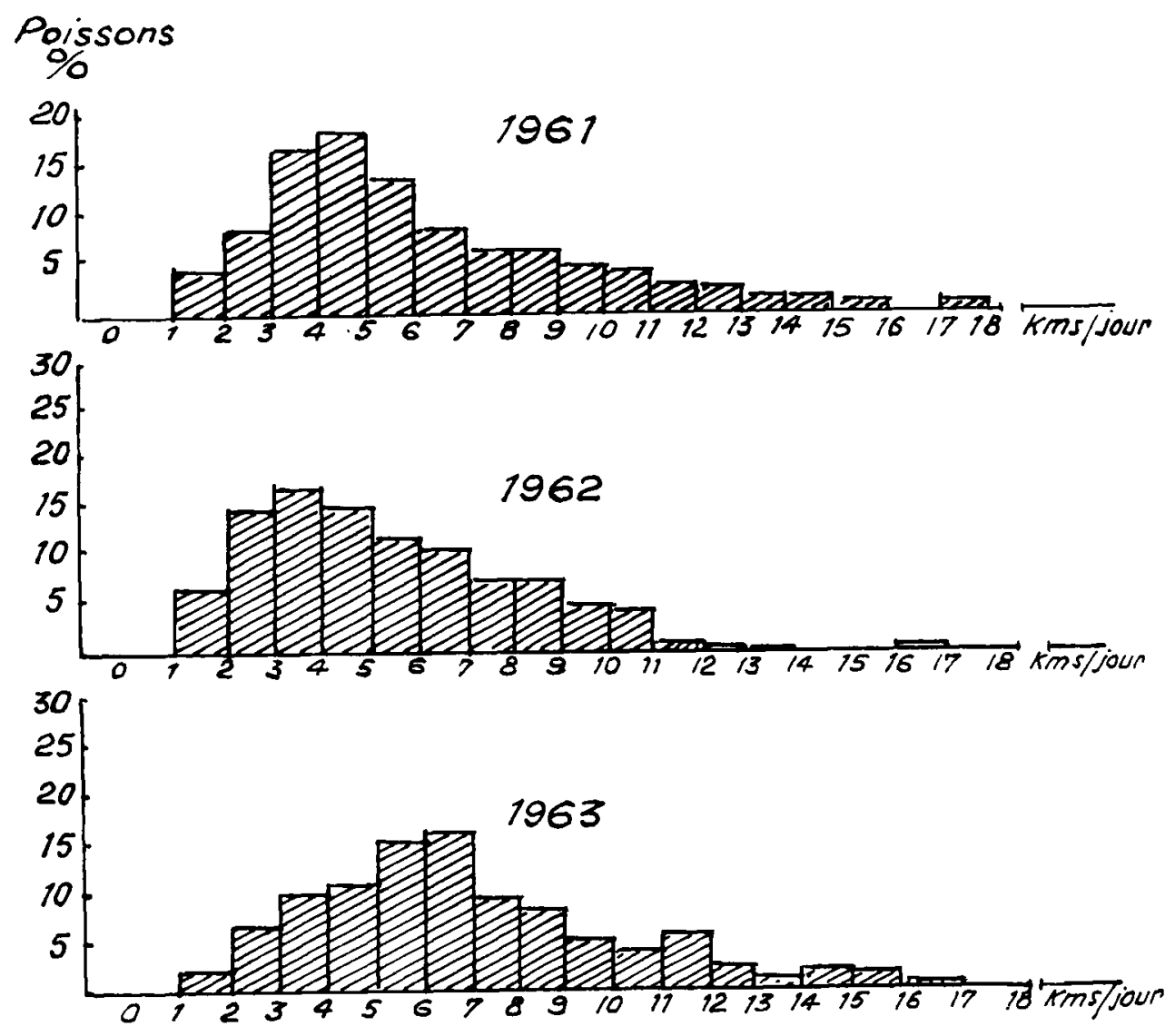

lïg. 3. - Vitesse de migration. 


\section{Influence des différents facteurs sur la vitesse.}

Dans cette étude on a voulu expliquer les causes de grande amplitude de la vitesse de migration; on a considéré l'influence de quatre facteurs : l'âge des poissons, le moment de la remontée dans la Vistule, l'état des organes sexuels et les prélèvements de nourriture pendant la migration.

L'âge des poissons et le moment de remontée sont sans influence sur la vitesse de migration. Mais l'état de maturité des organes sexuels et les conditions de nourriture pendant le voyage peuvent créer des différences sur cette vitesse. D'où la dispersion du troupeau en migration.

\section{CONCLUSIONS}

On peut dire :

1. Les poissons marqués sont représentatifs du troupeau migrateur mais avec les corrections citées dans ce travail.

2. La vitesse moyenne de migration $(6,4 \mathrm{~km}$ par jour) permet d'atteindre les frayères à $90 \%$ des poissons.

3. La population de géniteurs de Vimba vimba qui commence en automne sa migration vers les rivières des Carpathes constitue une seule population. 